\title{
Advances in Normal Conducting Accelerator Technology from the X-Band Linear Collider Program*
}

\author{
C. Adolphsen \\ Stanford Linear Accelerator Center, Stanford University, Stanford CA 94309
}

\begin{abstract}
In the mid-1990's, groups at SLAC and KEK began dedicated development of X-band $(11.4 \mathrm{GHz}) \mathrm{rf}$ technology for a next generation, TeV-scale linear collider. The choice of a relatively high frequency, four times that of the SLAC $50 \mathrm{GeV}$ Linac, was motivated by the cost benefits of having lower rf energy per pulse (hence fewer if sources) and reasonable efficiencies at high gradients (hence shorter linacs). To realize such savings, however, requires operation at gradients and peak powers much higher than that hitherto achieved. During the past twelve years, these challenges were met through innovations on several fronts. This paper reviews these achievements, which include developments in the generation and transport of high power rf, and new insights into high gradient limitations.
\end{abstract}

Presented at the 2005 Particle Accelerator Conference,

Knoxville, Tennessee, May 16-20, 2005

\footnotetext{
* Work supported by Department of Energy contract DE-AC02-76SF00515.
} 


\title{
ADVANCES IN NORMAL CONDUCTING ACCELERATOR TECHNOLOGY FROM THE X-BAND LINEAR COLLIDER PROGRAM*
}

\author{
C. Adolphsen \\ Stanford Linear Accelerator Center, Stanford University, Stanford CA 94309 USA
}

\begin{abstract}
In the mid-1990's, groups at SLAC and KEK began dedicated development of X-band (11.4 GHz) rf technology for a next generation, TeV-scale linear collider. The choice of a relatively high frequency, four times that of the SLAC $50 \mathrm{GeV}$ Linac, was motivated by the cost benefits of having lower rf energy per pulse (hence fewer rf sources) and reasonable efficiencies at high gradients (hence shorter linacs). To realize such savings, however, requires operation at gradients and peak powers much higher than that hitherto achieved. During the past twelve years, these challenges were met through innovations on several fronts. This paper reviews these achievements, which include developments in the generation and transport of high power rf, and new insights into high gradient limitations.
\end{abstract}

\section{INTRODUCTION}

The goal for a next generation electron-positron linear collider is to produce up to $1 \mathrm{TeV}$ center-of-mass collisions. During the past twelve years, SLAC and KEK worked to develop a collider design based on X-band $(11.4 \mathrm{GHz}) \mathrm{rf}$ technology for the main linacs. In 1999 , they were joined by FNAL, who contributed significantly to the accelerator structure development.

The linac rf system that was ultimately proposed uses $11.4 \mathrm{GHz}$ components similar in function to those in the 2.9 GHz SLAC Linac [1]. The baseline $\mathrm{rf}$ unit is illustrated in Figure 1. In each linac there would be about 1100 such units for $500 \mathrm{GeV}$ operation and twice that number for $1 \mathrm{TeV}$ operation. Each rf unit contains a modulator that drives two klystrons, each of which produces $75 \mathrm{MW}, 1.6 \mu \mathrm{s}$ rf pulses at $120 \mathrm{~Hz}$ or $150 \mathrm{~Hz}$. The klystrons power an $\mathrm{rf}$ compression system that produces higher peak power (by about three times) in shorter (four times) pulses that match the accelerator structure requirements. The resulting $400 \mathrm{~ns}, 475 \mathrm{MW}$ pulses feed eight, $0.6 \mathrm{~m}$ long accelerator structures, producing a $65 \mathrm{MV} / \mathrm{m}$ unloaded gradient in each structure. For efficiency, multiple bunches (192) are accelerated on each rf pulse. At the design bunch intensity $\left(0.75 \times 10^{10}\right.$ particles $)$ and bunch spacing ( $1.4 \mathrm{~ns})$, the beams $(0.86 \mathrm{~A})$ load the accelerator structures by $20 \%$, yielding a $52 \mathrm{MV} / \mathrm{m}$ loaded gradient.

The choice of rf frequency strongly influences the power source requirements. The $\mathrm{rf}$ attenuation time in the

\footnotetext{
* Work Supported by DOE Contract DE-AC03-76F00515.
}

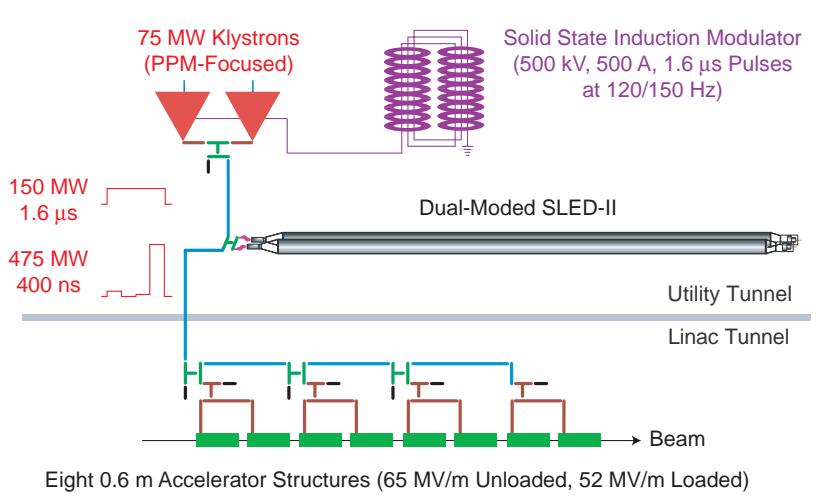

Figure 1: Schematic of a linac rf unit.

structures $(\sim 100 \mathrm{~ns})$ sets the scale for the structure fill time ( attenuation time) and the bunch train length ( 3 fill times) for good rf-to-beam efficiency and low cost. With the parameters chosen, the overall AC-to-beam energy transfer efficiency is about $8 \%$. When including the power for cooling and other purposes, $208 \mathrm{MW}$ of $\mathrm{AC}$ wall plug power would be required for both main linacs to generate $6.8 \mathrm{MW}$ average power beams for $500 \mathrm{GeV}$ operation.

In the following sections, the performance that has been achieved in prototypes of the major rf sub-systems is reviewed. With the recommendation by the ITRP last August that a next generation linear collider use superconducting rf technology, these normal conducting rf activities have been heavily curtailed (some close-out activities continue). The authors hope that the advances from the linear collider program will be useful in other applications.

\section{MODULATORS}

The basic requirement for the modulators is to power the $75 \mathrm{MW}$ klystrons, which each require $500 \mathrm{kV}, 270 \mathrm{~A}$, $1.6 \mu$ s pulses. Although this voltage is fairly high, it was not a major design constraint. The bigger challenges have been to achieve fast rise/fall times $(\sim 200 \mathrm{~ns})$ for efficiency, and to power several klystrons to reduce costs [2].

Conventional line-type modulators like those used in the SLAC Linac were considered initially for this application. These modulators have several drawbacks including low efficiency and their reliance on thyratrons, which have relatively short lifetimes (10-20 khr) and require periodic tuning. As an alternative, a solid-state induction-type modulator was developed by a SLAC, LLNL and Bechtel Nevada collaboration. The design takes advantage of recent advances in high-power, solid- 
state switches (Insulated Gate Bipolar Transistors or IGBTs), which are used primarily by the motor drive industry. The basic concept is to sum many low-voltage sources (few $\mathrm{kV}$ ) inductively to yield the desired klystron voltage. For this purpose, each source drives a toroidal transformer $(11 \mathrm{~cm}$ ID, $30 \mathrm{~cm}$ OD) made with Metglas $\AA$, an amorphous ferromagnetic metallic alloy. These cores are stacked and secondary windings are threaded through their IDs to sum the voltages. Each source is essentially a capacitor that is slowly charged and then partially discharged $(\sim 2 \%)$ through an IGBT switch to generate the pulse. Because of this limited discharge, much higher density capacitors are possible than in conventional modulators. To improve reliability, additional sources are included that automatically replace ones that fail. Overall, this design has the potential of being less expensive, easier to maintain and more efficient and reliable. Also, the pulse can be shaped by delaying turn-on of some of the sources.

For cost reasons, it is preferable to drive as many klystrons per modulator as possible. In an earlier rf system configuration, each rf unit contained eight klystrons that generated $75 \mathrm{MW}, 3.2 \mu$ s pulses. Given this layout, the first full-scale induction modulator was designed to drive eight such klystrons with about $1 \mathrm{GW}$ of peak power. A three-turn secondary was used with 76 transformers to match the IGBT ratings for pulsed operation (with two IGBTs per transformer, each ran at $2.2 \mathrm{kV}$ and $3.3 \mathrm{kA}$ ).

The development and construction of this 'Eight Pack' modulator took several years. Initial tests with a water load achieved $1 \mathrm{kA}$ pulses at $500 \mathrm{kV}$, and included 'spark down' evaluations where the output was shorted to simulate klystron gun arcs. Preventing IGBT failures from over-current in these cases required the adoption of a more robust IGBT design and the addition of feedback to the IGBT drive circuit to limit the current based on the rate of current change. These changes produced slower switching times and the increased IGBT heating that resulted limited the pulse rate. Tests also included driving one or two S-band klystrons at $400 \mathrm{kV}, 450 \mathrm{~A}$ each with $3 \mu \mathrm{s}$ pulses. Gun arcs were forced by increasing the voltage level. The modulator survived these events, as did the klystrons, even though much more energy was dissipated (up to $200 \mathrm{~J}$ ) than had been thought possible without damaging the guns.

The original plan was to use this modulator in an rf system test to power eight $75 \mathrm{MW}$ klystrons at the Next Linear Collider Test Accelerator (NLCTA). However, the klystron production was delayed and eventually the rf unit configuration was changed to that described above. So instead, four $50 \mathrm{MW}$ solenoid-focused tubes were installed, each of which required $300 \mathrm{~A}$ at $400 \mathrm{kV}$. Only one driver board was used per transformer in this case, which made operation easier as the power sharing between the two boards had not always been balanced. However, this did not help the IGBT heating problem, which was in fact made worse by the two-times higher stray capacitance of the $50 \mathrm{MW}$ tubes relative to that anticipated with the $75 \mathrm{MW}$ tubes. To allow $60 \mathrm{~Hz}$ operation, a chilled water system was used to cool the modulator. The increased stray capacitance also produced a $20 \%$ overshoot in the pulse, which was flattened by delaying the turn-on of some of the sources. With this procedure, a flattop of $+/-1 \%$ was achieved during the $1.6 \mu$ s long, $400 \mathrm{kV}$ pulses.

The modulator ran in this configuration for over 1500 hours at $60 \mathrm{~Hz}$, producing $400 \mathrm{kV}, 1200 \mathrm{~A}, 1.6 \mu$ s pulses. The efficiency from 'wall plug' to usable HV pulse was $58 \%$, while the original goal was $>75 \%$ (the higher stray klystron capacitance is the cause of some of this difference). One problem encountered during this period was failure of the bypass diodes that carry the current when the switch is off or delayed. These failures were due in part to the high turn-on voltage of the diodes. The problem was fixed by using an array of smaller, lower turn-on-voltage versions that were plugged into the unused board slots. A more persistent problem was failure of the multi-laminate connections between the boards and the transformer housing. The bad contacts led to overheating, arcing and intermittent board behavior, and required nearly daily maintenance to replace, clean or re-seat one or more of them.

Even before the Eight Pack modulator was first operated, a program was underway to improve its performance and lower its cost. The design that resulted has robust, spring-contact connectors, better current balance between boards, improved oil cooling, and faster, higher-voltage IGBTs (operated at $4 \mathrm{kV}$, so there are fewer, but thicker transformers). Also, a 'two-pack' layout was later adopted with the change to a twoklystron rf unit for the linear collider. To quickly demonstrate such a system, a hybrid modulator was configured in which 15 of the new transformers with dual-drive boards were stacked, and a single turn secondary wound through them to drive a conventional 10:1 turns-ratio transformer. This 'Two Pack Hybrid' was tested using a water load and produced $500 \mathrm{kV}, 500 \mathrm{~A}$, $1.6 \mu$ s pulses at $120 \mathrm{~Hz}$ with $60 \%$ efficiency (worsened by the conventional transformer). However, the cooling was still somewhat marginal and the IGBTs were not as robust as desired. Currently, this unit is being upgraded and will be installed in place of the Eight Pack transformer stacks to power two $50 \mathrm{MW}$ klystrons. A fully integrated Two Pack modulator was designed but will not be built. However, research continues to develop smaller IGBT modules with integrated gate drivers.

\section{KLYSTRONS}

To reduce the power source cost, it is desirable to use high peak power klystrons. Such klystrons are challenging to design because they require a high-voltage, largearea gun, a strong beam focusing system with both low power consumption and low beam loss, a stable, highgradient output section and robust, high power vacuum 
windows. In general, pulse lengths longer than the $400 \mathrm{~ns}$ needed for the accelerator structures are possible, so rf pulse compression is employed to utilize the available energy. Such schemes are cost effective for pulse lengths from about $1.2 \mu$ s to $3.2 \mu \mathrm{s}$, but with the longer pulses, achieving adequate klystron cooling is a challenge.

The first tubes developed used conventional solenoid focusing, although the associated power dissipation $(20 \mathrm{~kW})$ was too high to be practical for the large number of tubes required for the linear collider. At SLAC, the 50 MW XL4 klystrons were the culmination of this program, which started with a goal of producing $100 \mathrm{MW}$ tubes. The XL4 klystrons have a micro-perveance of 1.2 and contain four-cell, traveling-wave output cavities. Twelve have been built and they have been used extensively for component testing and beam acceleration where they produce up to $50 \mathrm{MW}, 1.6 \mu$ s pulses at $60 \mathrm{~Hz}$ with a $41 \%$ efficiency. At KEK, a similar tube was developed with BINP and it produced $50 \mathrm{MW}, 1.5 \mu \mathrm{s}$ pulses with $35 \%$ efficiency.

To eliminate the power consuming solenoid electromagnets, a program at SLAC was started in 1995 to develop periodic permanent magnet (PPM) focusing. The goal for these PPM klystrons has been to produce $75 \mathrm{MW}$ in up to $3.2 \mu$ s pulses with better than $55 \%$ efficiency. In the designs that resulted, about 40 magnet rings with alternating polarities are interleaved with iron pole pieces to generate a sine-like axial field along the $\sim 0.5 \mathrm{~m}$ long, $\sim 10 \mathrm{~mm}$ diameter beam tube between the gun anode and beam collector. The resulting focusing strength is proportional to the rms of this axial field, and about $2 \mathrm{kG}$ can be achieved practically compared to the $\sim 5 \mathrm{kG}$ field in the solenoid-focused tubes. This has led to a lower microperveance (0.8) design in which the space charge defocusing is smaller. This choice requires a fairly high voltage gun $(500 \mathrm{kV})$, but increases the klystron efficiency due to the improved bunching. For the magnets, $\mathrm{NbFeB}$ has been mainly used because of its low cost. To maintain field quality, the magnet temperature must be kept below about $50{ }^{\circ} \mathrm{C}$.

To date, groups at SLAC and at KEK (in collaboration with Toshiba) have each designed and built seven PPM klystrons [3,4]. The learning curve has been steep as numerous problems have plagued the tubes after initial success was achieved with $50 \mathrm{MW}$, low average power prototypes. At SLAC, the development was also complicated by design changes introduced to improve the tube manufacturability. The problems have included gun arcing and oscillations, poor quality magnets, magnet misalignments, output cavity and collector oscillations and rf breakdown in the output section and at the windows. These problems have been mostly overcome through design and manufacturing improvements. For example, rf absorber material was added in the SLAC gun to suppress a $1.4 \mathrm{GHz}$ oscillation.

At SLAC, two tubes have met the full spec of $75 \mathrm{MW}$, $1.6 \mu$ s operation at $120 \mathrm{~Hz}$ with $54 \%$ efficiency. The beam transmission in the most recent tube was over $99 \%$ (some beam loss is avoidable during the HV turn on/off period). The KEK/Toshiba collaboration has produced four tubes that generated $75 \mathrm{MW}, 1.6 \mu$ s pulses with similar efficiencies, but at $50 \mathrm{~Hz}$ due to modulator limitations. During the last six months, however, one of the earlier problems has been found to be more endemic. All six of the above mentioned tubes suffer chronic rf breakdown in the output section. These events are characterized by a loss of transmitted power that develops over several hundred ns without a loss in beam transmission. The breakdowns are likely occurring in the last output cell since the transmitted power from the two output ports are typically asymmetric (although the power goes to zero in each case). The rate of such events becomes progressively worse over time and does not improve using standard if processing techniques. Such events were also seen in two XL4 klystrons operating at $50 \mathrm{MW}$ after exposure to reflected power from hundreds of breakdowns in downstream waveguide. In addition, breakdown occurred at a low rate in the $50 \mathrm{MW}$ PPM tubes.

At $75 \mathrm{MW}$, the peak surface field on the last output cavity iris is about $70 \mathrm{MV} / \mathrm{m}$. This is far below that estimated to be sustainable $(\sim 110 \mathrm{MV} / \mathrm{m}$ at $1.6 \mu \mathrm{s})$ from the experience with low group velocity $(\sim 3 \% \mathrm{c})$ accelerator structures (discussed below). However, these fields exceed the maximum values achieved $(\sim 50 \mathrm{MV} / \mathrm{m}$ at $1.5 \mu \mathrm{s})$ in a test of a waveguide with group velocity similar to that $(10-20 \%)$ in the traveling-wave klystron output sections [5].

The effort to understand the origin of these events continues, including the sectioning of the output regions of some of the tubes. Up to four new tubes will be tested in the next year, which may yield further clues. If the breakdown problem proves to be fundamental, a sheetbeam or multi-beam klystron approach may be required to produce such high power stably.

\section{RF DISTRIBUTION}

The rf distribution system connecting the klystrons to the structures includes a pulse compressor that shortens the pulse while increasing the peak power. In early rf system designs, a SLED II pulse compression scheme was proposed, which is a delay line version of the SLAC Linac Energy Doubler (SLED). It includes a hybrid that splits the klystron power and routes each half to a delay line made of circular waveguide. These lines are shorted at the far end and have irises at the near end that partially reflect the rf. During operation, the lines are resonantly filled and then effectively discharged by a $180^{\circ}$ change of the klystron rf phase. Three lower-power SLED II systems have performed well at NLCTA, with over 30,000 hours of $60 \mathrm{~Hz}$ operation up to $250 \mathrm{MW}$. However, they are not particularly efficient $(65 \%)$ due to the rf that is transmitted from the delay lines during filling. 
While more efficient compression methods were considered for the linear collider, SLED II was eventually chosen because of its relative simplicity, which also made it easier to demonstrate (e.g., the Delay Line Distribution System alternative would have required eight klystrons). The basic SLED II requirements are to produce $475 \mathrm{MW}$, $400 \mathrm{~ns}$ pulses with $81 \%$ efficiency. The version proposed for the linear collider differs from the early NLCTA design in several ways, including larger diameter $(17.1 \mathrm{~cm})$ delay lines to lower power loss, dual-moded (TE01 and TE02) delay line transport to halve the delay line length, a lower compression ratio (4 instead of 6 ) to improve efficiency, and the use of over-height planar hybrid and waveguide components to lower surface fields. A design surface field limit of $50 \mathrm{MV} / \mathrm{m}$ was chosen based on tests of waveguide and a high-power SLED II prototype [5,6].

A prototype dual-moded SLED II system for the linear collider was built at NLCTA in 2003 as part of the Eight Pack Project [7]. After high-power processing this system for three weeks, $580 \mathrm{MW}, 400 \mathrm{~ns}$ pulses were achieved, limited only by the available klystron power. The system was then run at $500 \mathrm{MW}$ for 200 hours at $30 \mathrm{~Hz}$ and 100 hours at $60 \mathrm{~Hz}$ to measure its performance. During the $30 \mathrm{~Hz}$ operation, $11 \mathrm{rf}$ breakdowns occurred in the SLED II system and associated over-height waveguide, while none occurred during the $60 \mathrm{~Hz}$ operation. The average rate of 0.06 breakdowns per hour $(60 \mathrm{~Hz}$ equivalent) is better than the 0.16 per hour limit for the linear collider, and the improvement during the last 100 hours suggests that the breakdown rate was decreasing. The efficiency achieved after optimal tuning was $77 \%$. This system was subsequently run at $300 \mathrm{MW}$ for over 1500 hours for structure testing. It has yet to be fully characterized as to its power handling capability.

\section{ACCELERATOR STRUCTURES}

The basic structure design chosen for the linear collider is traveling wave with a disk-loaded waveguide geometry. As is typical in such designs, the rf group velocity is varied along the structure to produce a fairly constant surface gradient profile, with or without including the effect of beam loading. The choice of operating gradient was based in part on cost optimization studies that considered the relative cost of the power sources (which increase with gradient) and the beam line (which decreases with gradient). The design unloaded gradient of $65 \mathrm{MV} / \mathrm{m}$ is less than the cost optimum ( $80 \mathrm{MV} / \mathrm{m})$, but the cost difference is only a few percent of the collider cost. Another important consideration was the practical gradient that could be achieved, which required a major R\&D effort to quantify.

The structure gradients are limited by rf breakdown and the surface damage it causes. While the breakdown rate strongly depends on the surface field strength, the damage that results (i.e., the pitting on the iris surfaces) appears to depend on the incident power level. At high power, this damage leads to breakdown on subsequent pulses, which ultimately limits the achievable gradient. With this realization, the design efforts focused on reducing the input power, which is complicated by efficiency and wakefield constraints.

The strength of both the short-range (inter-bunch) and long-range (bunch-to-bunch) structure wakefield must be limited to preserve the small beam emittances in the linear collider. For long-range wakefield suppression, a combination of dipole mode damping and detuning was implemented, which required innovations in wakefield modeling and in quality control during cell fabrication. The detuning was achieved by tailoring the cell dipole frequencies to produce a Gaussian-like distribution, and damping is provided by four manifolds that run parallel to the structure and couple to each cell. These methods were shown to produce a hundred-fold reduction in the longrange wakefield by the arrival time of the next bunch (1.4 ns), as is required for the linear collider [8].

The short-range wakefield limit tightly constrains the allowed minimum average iris radius, which would otherwise be reduced to improve the rf-to-beam efficiency. The average iris radius finally adopted is $17 \%$ of the rf wavelength. This decision was also tied to the choice of bunch charge, and a less than optimal rf-to-beam efficiency ( $48 \%$ in steady state compared to $\sim 70 \%$ maximum with the final structure design) was accepted as a tradeoff for smaller wakefields. With these choices, the linac beams must be centered within the structures to about $10 \mu \mathrm{m}$ rms, which has been demonstrated using the dipole signals from the damping manifolds as an alignment guide [9].

An aggressive program to establish stable high gradient operation of the structures began in 1999 when it was realized that the long $(1.8 \mathrm{~m})$, high-power designs that had been developed to optimize the cost of the collider performed much worse than the short, low-power, highwakefield designs that had been developed initially for expediency. Since then, 40 structures have been tested at NLCTA with over 25,000 hours of high power operation logged $[10,11]$. The viable structure designs that have resulted from this program are $60 \mathrm{~cm}$ long and have low group velocity (3-4\% c initially) and high phase advance per cell $\left(150^{\circ}\right)$. Relative to the $1.8-\mathrm{m}$ structures, they are $16 \%$ less efficient, but have vastly improved performance at the gradients of interest with no indication of significant damage after initial processing to about $75 \mathrm{MV} / \mathrm{m}$ (typically $<2^{\circ}$ change in net phase advance).

During the past year and half, this program has focused on quantifying the structure performance. With the large number of structures required $(\sim 18,000)$ for the linear collider, the structure trip rate from rf breakdown must be very small $(<0.1$ per hour at the $60 \mathrm{~Hz}$ rate used when testing) to ensure essentially $100 \%$ full linac energy availability with the planned $2 \%$ overhead of spares and a 10 second trip recovery time. Also, the transverse fields generated during breakdown would deflect the beam so 
the magnitude of these kicks must be small. Finally, the structures need to be robust in that they do not degrade in performance over time, and recover quickly if accidentally vented to air.

To measure the long-term performance, eight structures were operated at $65 \mathrm{MV} / \mathrm{m}$ (unloaded) with the design pulse shape (100 ns amplitude ramp plus a $300 \mathrm{~ns}$ flattop). Of these structures, five have all the wakefield suppression features required for the linear collider. After more than 1500 hours of $60 \mathrm{~Hz}$ operation, the structure breakdown rates ranged from 0.001 to 0.07 per hour, with an average of 0.04 per hour, which is well within the 0.1 per hour goal. During this period, the rates generally decreased exponentially with time constants of 400-700 hours. In the linear collider, the rates would likely be even lower due to the field reduction from beam loading.

In a dedicated test, the beam kicks caused by the fields generated during breakdown were measured and found to be large enough ( $10 \mathrm{keV} / \mathrm{c} \mathrm{rms})$ to cause some luminosity loss from off-center beam collisions in the linear collider $(<1 \%$ loss at the 0.1 per hour breakdown rate limit), but not large enough to cause the beams to hit the collimators that would be used. Another test showed that the structures could run without a recovery period after breakdown when the $\mathrm{rf}$ is normally shut off and then ramped back up. Finally, a series of venting experiments were conducted on a pair of previously processed structures to determine the influence of nitrogen and air exposure on performance. Even with exposure to unfiltered air, the low breakdown rates were recovered after a few days of operation during which a few dozen breakdowns occurred. Thus, the structures meet the linear collider requirements and appear to be robust.

Currently, measurements of wakefield suppression and beam-based structure alignment are underway to verify that the $60-\mathrm{cm}$ structures perform as well as the $1.8-\mathrm{m}$ structures in these regards [12]. Also, some high gradient testing continues at SLAC and KEK [13].

\section{SUMMARY}

The X-band linear collider program made significant advances in pulsed $\mathrm{HV}$ and $\mathrm{rf}$ power generation, high gradient acceleration and wakefield suppression. For the rf systems, a major constraint was surface field limits, which appear to depend on the energy flow through the devices. From tests of X-band traveling-wave structures, waveguides and single cell cavities, the general trend observed in sustainable surface fields is plotted in Figure 2 versus group velocity (or equivalent), which characterizes the energy flow in these systems (the field limits are shown for $250 \mathrm{~ns}$ pulses: they scale roughly as the $-1 / 6$ to $-1 / 3$ power of pulse length). For the linear collider, the structure design evolved to be within these limits, while the waveguide systems were initially. If $\mathrm{rf}$ breakdown proves to be a fundamental limit to stable operation of the $75 \mathrm{MW}$ klystrons, it would be consistent with these data. Thus, future accelerator designers should be wary of this trend with group velocity.

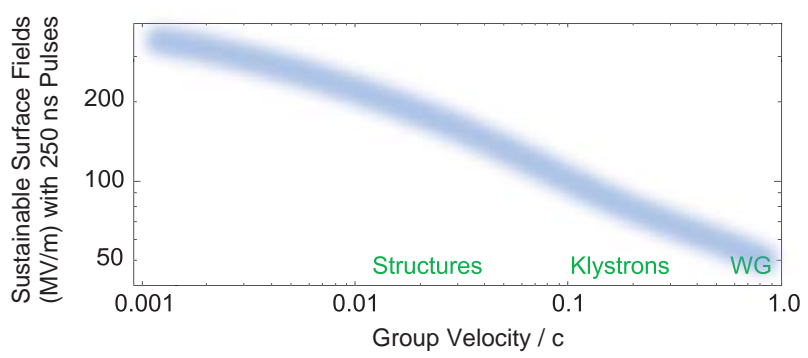

Figure 2: Trend of sustainable surface fields versus group velocity in various structures, waveguides and cells for pulse lengths of $250 \mathrm{~ns}$. The range of group velocities for the linear collider components are indicated by 'Structures,' 'Klystrons' and 'WG.'

\section{REFERENCES}

[1] "U.S. Linear Collider Technology Options Study," http://www.slac.stanford.edu/xorg/accelops (2004).

[2] R. Cassel et al., "NLC Hybrid Solid State Induction Modulator," LINAC 2004 (Aug. 2004).

[3] D. Sprehn et al., "Current Status of the Next Linear Collider X-Band Klystron Development Program," SLAC-PUB-11162 (July 2004).

[4] Y. H. Chin, "Status of the GLC X-Band Power Source R\&D," PAC 2003 (May 2003).

[5] V. Dolgashev et al., "RF Breakdown in X-band Waveguides," SLAC-PUB-10355 (June 2002).

[6] S. Tantawi et al., "The Generation of 400-MW RF Pulses at X-Band Using Resonant Delay Lines," IEEE Transactions on Microwave Theory and Techniques, p. 2539, Vol. 47, No. 12 (Dec. 1999).

[7] S. Tantawi et al., "Experimental Demonstration of an RF System for the X-band Linear Collider," SLAC-PUB-10550 (March 2004).

[8] C. Adolphsen et al., "Measurement of Wakefield Suppression in a Damped and Detuned X-Band Accelerator Structure," SLAC-PUB-7519 (May 1997).

[9] C. Adolphsen et al., "Wakefield and Beam Centering Measurements of a Damped and Detuned X-Band Accelerator Structure," SLAC-PUB-8174 (Sept. 1999).

[10] C. Adolphsen et al., "Normal Conducting RF Structure Test Facilities And Results," SLAC-PUB9906 (Sept. 2003).

[11] S. Doebert et al., "High Gradient Performance of Prototype NLC/GLC X-Band Accelerator Structures," these proceedings.

[12] S. Doebert et al., "Beam Position Monitoring Using HOM-Signals from a Damped and Detuned Accelerator Structure," these proceedings.

[13] T. Higo et al., "High Gradient Study at KEK on X-Band Accelerator Structure for Linear Collider," these proceedings. 